\title{
DIE AUSWIRKUNGEN DER COVID-19-PANDEMIE AUF DIE KREBSDIAGNOSE IN EINEM PATHOLOGIEDIENST IN SÜDBRASILIEN
}

\section{ORIGINALER ARTIKEL}

KLOCK, Julia Cristhina Monteiro¹, Borges, Giuliano Santos², OGATA, Daniel Cury ${ }^{3}$, KLOCK, Clóvis ${ }^{4}$

KLOCK, Julia Cristhina Monteiro. Et al. Die Auswirkungen der Covid-19-Pandemie auf die Krebsdiagnose in einem Pathologiedienst in Südbrasilien. Revista Científica Multidisciplinar Núcleo do Conhecimento. Jahr. 06, Hrsg. 11, Vol. 14, S. 182 -190. Oktober 2021. ISSN: 2448-0959, Zugangslink: https://www.nucleodoconhecimento.com.br/gesundheit/pathologiedienst, DOI: 10.32749/nucleodoconhecimento.com.br/gesundheit/pathologiedienst

\section{ZUSAMMENFASSUNG}

Die Covid-19-Pandemie hat erhebliche Auswirkungen auf das Leben der Menschen. Eine dieser Auswirkungen kann bei der Diagnose anderer Krankheiten, insbesondere von Krebs, nachgewiesen werden. Problem: Welche Auswirkungen hatte die Pandemie auf die Diagnose neuer Krebsfälle in der Region, die von einem Pathologiedienst in Südbrasilien abgedeckt wurde? Allgemeines Ziel: Es sollte untersucht werden, ob die Zahl der Krebsdiagnosen in einem Pathologiedienst in Südbrasilien während der Pandemie zurückgegangen ist. Methodik: Wir analysierten die Fallberichte, die in den Labors der Infolaudo-Gruppe in den ersten Monaten der Pandemie (April und Mai 2020) durchgeführt und mit den gleichen Monaten des Jahres 2019 verglichen wurden, um die Auswirkungen der Pandemie auf die

\footnotetext{
${ }^{1}$ Medizinstudent - Universität Mogi das Cruzes. ORCID: 0000-0002-0834-193X.

2 Spezialität in Onkologie, Abschluss in Medizin. ORCID: 0000-0002-0737-7922.

${ }^{3}$ Doktor der Chirurgie, Master in Chirurgie, Spezialität in Pathologie, Abschluss in Medizin. ORCID 0000-00018819-2155.

${ }^{4}$ Doktorand in Onkologie, Spezialis
}

RC: 103132

Verfügbar in: 
Diagnose von Krebs in einem Pathologiedienst in Südbrasilien zu messen. Ergebnisse: Vergleicht man die Zeiträume April und Mai 2019 mit dem gleichen Zeitraum im Jahr 2020, so wurde ein Rückgang der pathologischen Eingriffe um $34,2 \%$ festgestellt. Einige Krebsarten, wie Dickdarm, Speiseröhre, Brust und Prostata, hatten eine signifikante Abnahme der Diagnosen, die 19,7\%, 45\%, 18,2\% bzw. 37,7\% betrugen. Fazit: Mit der Schließung vieler Kliniken und Kliniken in den frühen Stadien der Pandemie kam es zu einem Rückgang der Krebsdiagnosen in den befragten Laboren.

Schlüsselwörter: Pandemie, Covid 19, Krebs, Pathologie.

\section{EINLEITUNG}

Die Weltgesundheitsorganisation (WHO) schätzt, dass es bis 2030 weltweit 27 Millionen neue Krebsfälle, 17 Millionen Todesfälle durch die Krankheit und 75 Millionen Menschen mit Krebs geben wird (INCA, 2020).

Krebs ist die häufigste Todesursache in Industrie- und Entwicklungsländern (ALLGAR und NEAL, 2005). Bestimmte Krebsarten haben jedoch eine hohe Heilungschance, wenn sie frühzeitig erkannt und angemessen behandelt werden (AMBUSAIDI und AL-BALUSHI, 2012).

Verzögerungen bei der Diagnose von Krebs können während der gesamten Diagnose auftreten, je nach den Ebenen der Gesundheitsversorgung: Patient, Grundversorgung und Sekundärversorgung.Diagnosen bei fortgeschritteneren neoplastischen Erkrankungen können auftreten, wenn der Patient in Bezug auf vermutete Symptome nur langsam erkennt und handelt (ASCO, 2020). Dieses geringe Bewusstsein für frühe Symptome von Krebs gilt als der vorherrschende Grund für eine späte Präsentation, insbesondere wenn die Symptome atypischer Natur sind (ASCO, 2020). Ein weiteres mögliches Hindernis kann mit der hohen Nachfrage nach spezialisierten medizinischen Dienstleistungen zusammenhängen 
und schließlich die Diagnose verzögern, insbesondere im öffentlichen Dienst (INCA, 2020).

In Brasilien wurden laut the INCA im Jahr 2020 schätzungsweise 309.230 neue Krebsfälle geschätzt, bei Männern war Prostatakrebs für 29,2\% der geschätzten Fälle verantwortlich. Bei Frauen entsprach Brustkrebs von den 316.140 erwarteten neuen Fällen 29,7\%. Diese Daten beinhalten keinen Nicht-Melanom-Hautkrebs (GREEN et al., 2015).

In einem Fall kann die Verzögerung der Krebsdiagnose auf mehreren Ebenen auftreten. Der Patient kann vermutete Symptome von Krebs nicht erkennen oder auf sie reagieren. Der Hausarzt erkennt möglicherweise Patienten mit Verdacht auf Krebssymptome nicht und untersucht sie nicht ordnungsgemäß oder überweist sie rechtzeitig. Patienten mit klinischem Verdacht in der Sekundärversorgung werden möglicherweise nicht rechtzeitig gesehen oder an die falsche Spezialität überwiesen.

Während der Coronavirus-Pandemie wirkten sich die ergriffenen Präventionsmaßnahmen wie Social Distancing und Quarantäne auf die Erstdiagnose von Krebspatienten aus. So machte es die in mehreren Krankenhäusern beobachtete Überbelegung unmöglich, diese Patienten richtig zu diagnostizieren und zu behandeln.

In diesem Szenario fehlen Daten aus der lokalen Stichprobe, um zu überprüfen, ob die Pandemie diese Patientengruppe und Krebsdiagnosen wirklich betroffen hat.

Vor diesem Hintergrund hat der vorliegende Artikel als Leitfrage: Welche Auswirkungen hatte die Pandemie auf die Diagnose neuer Krebsfälle in der Region, die von einem Pathologiedienst in Südbrasilien abgedeckt wurde? Mit dem allgemeinen Ziel, zu bewerten, ob die Zahl der Krebsdiagnosen in einem Pathologiedienst in Südbrasilien während der Pandemie zurückgegangen ist.

Daten vom Beginn der Pandemie (April und Mai) von 2020 wurden analysiert, verglichen mit Daten von April und Mai 2019, von einem Pathologiedienst der

RC: 103132

Verfügbar in:

https://www.nucleodoconhecimento.com.br/gesundheit/pathologiedienst 
südlichen Region Brasiliens (Infolaudo-Gruppe), um die aktuelle Situation und die Auswirkungen der Lockdown-Phase auf die Diagnose dieser Personengruppe zu erkennen.

Hierbei handelt es sich um eine epidemiologische Querschnittsstudie der lokalen Stichprobe.

\section{MATERIALIEN UND METHODEN}

Alle Fallberichte vom April und Mai 2020 und April und Mai 2019 wurden analysiert, in den Labors der Infolaudo-Gruppe durchgeführt und miteinander verglichen. Die gewählten Zeiträume wurden aufgrund des Beginns der sozialen Isolation und der Lockdown-Maßnahmen definiert. Alle in diese Studie eingeschlossenen Patienten wurden in der Foz-Region des Flusses Itajaí, im Alto Vale, im Großraum Florianópolis und im Norden und Westen des Rio Grande do Sul (Erechim und Cruz Alta) behandelt.

Alle Fälle, bei denen Krebs diagnostiziert wurde, wurden in die Studie einbezogen. Fälle, die keine Bestätigung der Krebsdiagnose hatten, wurden ausgeschlossen. Die gesammelten Daten flossen in das Microsoft Office Excel®-Programm ein 2010 und die Ergebnisse wurden in absoluten Zahlen und Prozentsätzen dargestellt. Hierbei handelt es sich um eine epidemiologische Querschnittsstudie der lokalen Stichprobe.

\section{DISKUSSION}

Verzögerungen in der Primärversorgung können bei der Erkennung, Untersuchung und Überweisung von Fällen mit Verdacht auf maligne Neoplasie auftreten (ASCO, 2020).Obwohl die Primärversorgung in mehreren Ländern der Welt die erste Anlaufstelle für Patienten ist, die nationale Gesundheitsdienste in Anspruch nehmen, bleibt die Verzögerung der Krebsdiagnose auf dieser Ebene ein anhaltendes Problem (JONES et al., 2007).Von Hausärzten wird erwartet, dass sie Patienten mit einer möglichen Erkrankung frühzeitig identifizieren. Die Diagnose Krebs ist jedoch 
für den Hausarzt relativ selten, da mehr als $80 \%$ der Patienten unspezifische Symptome haben (HARFORD, 2011). In der Tat besteht das Hauptanliegen dieser Angehörigen der Gesundheitsberufe darin, die Minderheit der Patienten, die dringend Aufmerksamkeit benötigen, von denen zu unterscheiden, die wahrscheinlich an selbstbegrenzten Erkrankungen leiden (KAUFMAN et al., 2020).

Im Mai 2020 veröffentlichte die American Society of Clinical Oncology (ASCO) einen Sonderbericht, in dem die Verschiebung von Klinikbesuchen und Krebsvorsorgeuntersuchungen oder Diagnose- und Staging-bezogenen Verfahren empfohlen wird, wenn diese Verschiebung kein Risiko für ein Fortschreiten der Krankheit oder eine Verschlechterung der Prognose darstellt (LONDON et al., 2020).

Einige internationale Studien zeigen, dass der Rückgang der Krebsdiagnose in den ersten Monaten der Pandemie 65,2\% der neuen Krebsfälle betrug (LONDON et al., 2020).

Das screening einiger Krebsarten war beeinträchtigt, wobei Die Daten zeigten, dass Brust-, Dickdarm- und Rektumkrebs mit 89,2 bzw. 84,5\% am stärksten betroffen waren (MACLEOD et al., 2009).

In einer in Großbritannien durchgeführten Studie führte der lockdown zur Aussetzung von Krebsvorsorgeuntersuchungen, was die Früherkennung unzähliger Patienten gefährdete. Nur davon wurden Patienten mit kritischem und symptomatischem Krankheitsbild auf diagnostische Interventionen angewiesen. Krebsaufzeichnungen wurden im National Health Service (NHS) über eine Datenbank von Krankenhäusern mit Patienten im Alter von 15 bis 84 Jahren verwendet, bei denen Brustkrebs (35583), Darmkrebs (24975) und Speiseröhrenkrebs (6744) im Jahr 2010 mit Followup bis 2014 diagnostiziert wurde. Bei Patienten mit primärer Lungenneoplasie (29305) wurde 2012 als Jahr der Diagnose und 2015 als Enddatum der Nachbeobachtung verwendet. Durch ein Flussdiagramm zur Definition der Wege von Krebspatienten innerhalb des NHS wurde eine Schätzung vorgenommen, um die Folgen der diagnostischen Verzögerung in dieser Patientengruppe über einen 
Zeitraum von 12 Monaten ab März 2020 (Datum des Lockdowns) zu bewerten und die Auswirkungen 1, 3 und 5 Jahre nach der Erstdiagnose zu kontextualisieren. Bei dieser Methodik wurden drei Wege oder Ströme dieser Patienten betrachtet, die vom besten bis zum schlimmsten Szenario entsprechen. Auf dieser Grundlage wurden die tatsächlichen Auswirkungen des Überlebens 1, 3 und 5 Jahre nach der Diagnose geschätzt, wobei die Gesamtzahl der durch Krebs verursachten Todesfälle und die Gesamtzahl der verlorenen Lebensjahre im Vergleich zu den Daten vor der Pandemie berechnet wurden.

In allen Szenarien wurde innerhalb von 5 Jahren nach der Diagnose ein Anstieg der Todesfälle durch Brustkrebs um 7,9\% bis 9,6\% geschätzt, was 281 bis 344 weitere Todesfälle bedeutet. Bei Darmkrebs betrug der Anstieg von 15,3\% (1445) auf 16,6\% (1563) und bei Lungenkrebs betrug dieser Anstieg 4,8\% (1235) bis 5,3\% (1372). Schließlich betrug der bei Patienten mit Speiseröhrenkrebs beobachtete Anstieg 5,8\% (330) bis 6\% (342). Diese Daten zeigen, dass es im Vereinigten Königreich einen signifikanten Anstieg vermeidbarer Todesfälle gegeben hat, wahrscheinlich aufgrund restriktiver und sozialer Isolationsmaßnahmen (MARINGE et al., 2020).

Eine weitere studie, die von Kaufman et al. (2020) veröffentlicht wurde, beobachtete seit Beginn der Covid-19-Pandemie eine Verringerung der wöchentlichen Krebsinzidenz in den Niederlanden um 40\% und im Vereinigten Königreich um 75\%. Diese Studie verwendete eine ähnliche Methodik wie wir und bewertete die Datensätze einer Datenbank von Januar bis April 2019 im Vergleich zum gleichen Zeitraum des Jahres 2020. Sie bemerkten signifikante Rückgänge bei der Diagnose von bösartigen Neubildungen, in situ und unbestimmtem Verhalten. Während der Pandemie sank die wöchentliche Zahl der Fälle bei den sechs häufigsten Krebsarten um mehr als $46 \%$, mit Variationen von $24,7 \%$ bei Pankreasneoplasie und $51,8 \%$ bei Brustfällen (WHO, 2016).

Ein weiterer Artikel, der von Forschern des Sidney Kimmel Center in Philadelphia und des NHS veröffentlicht wurde, zeigte eine Verringerung des Brustkrebs- 
Screenings um 89,2\% und eine Abnahme der Koloskopie um 85,5\%, was ein wichtiges Instrument zur Früherkennung von Darmkrebs ist (VOSE, 2020).

Alle Fälle der Infolaudo-Gruppe in den Zeiträumen März und April 2020 wurden mit dem Zeitraum März und April 2019 verglichen. Die Monate März und April wurden ausgewählt, da es sich um die ersten Monate des Lockdowns handelte.

Im Jahr 2019, im April und Mai, erhielten die Laboratorien der Infolaudo-Gruppe 5524 pathologische Eingriffe, von denen 4634 gutartige Läsionen und 890 maligne Läsionen waren, was $16,1 \%$ entspricht. Im Jahr 2020, im gleichen Zeitraum, gab es 3635 Eingriffe mit 2.875 gutartigen Läsionen und 760 malignen Läsionen, was bösartigen 20,9\% entspricht. Es gab eine Abnahme der pathologischen Verfahren um $34,2 \%$. (Schaubild 1)

Abbildung 1: Rückgang der pathologischen Eingriffe im April und Mai 2020 im Vergleich zum gleichen Zeitraum 2019.

\section{Lesões benignas X Lesões malignas}

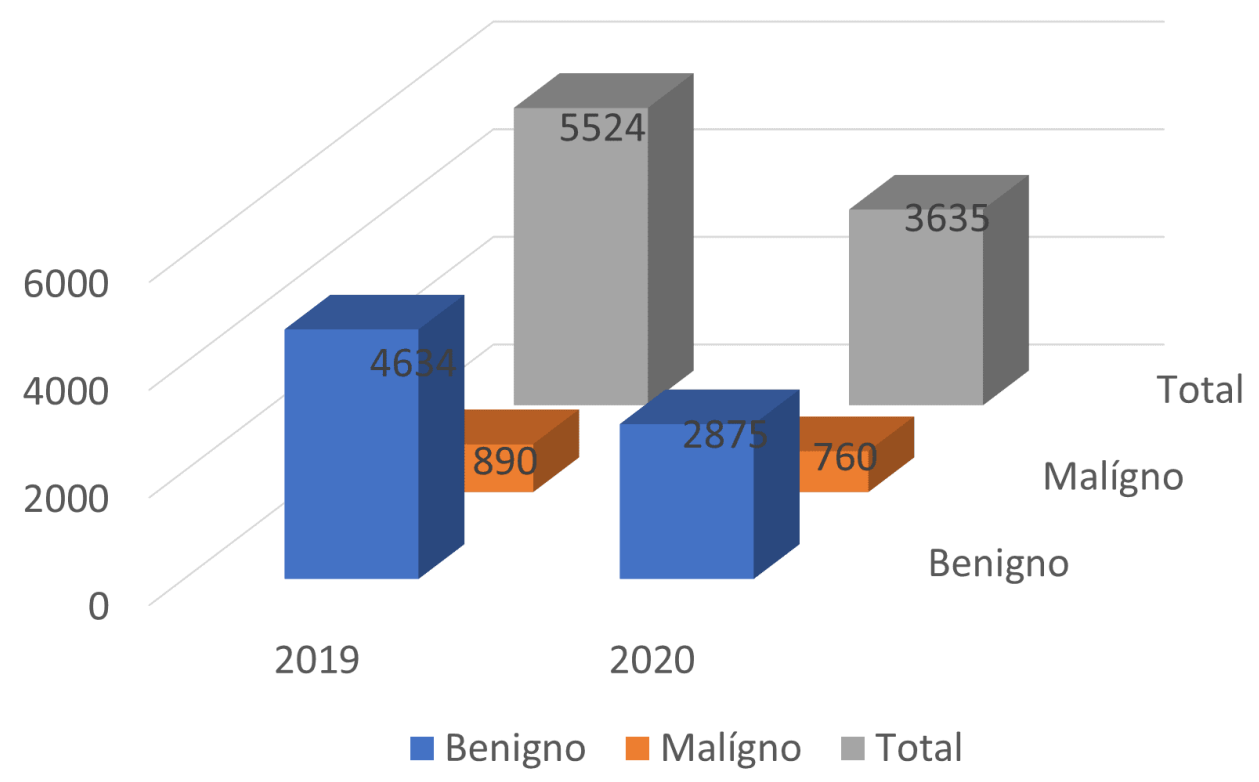

Quelle: Autoren

RC: 103132

Verfügbar in:

https://www.nucleodoconhecimento.com.br/gesundheit/pathologiedienst 
Im Allgemeinen gab es bei allen verschiedenen Verfahren eine Abnahme der Diagnose, seien es Biopsien oder chirurgische Proben. Einige Arten von Verfahren hatten einen deutlicheren Rückgang, wie z.B. größere elektive Operationen, die einen Krankenhausaufenthalt des Patienten erforderten.

Einige Krebsarten (Grafik 2) wiesen einen signifikanteren Rückgang auf, wie Dickdarm (19,7\%), Speiseröhre (45\%), Brust $(18,2 \%)$ und Prostata $(37,7 \%)$.

Abbildung 2: Rückgang der am stärksten betroffenen Krebsdiagnosen (nach Organen) im April und Mai 2020 im Vergleich zum gleichen Zeitraum 2019.

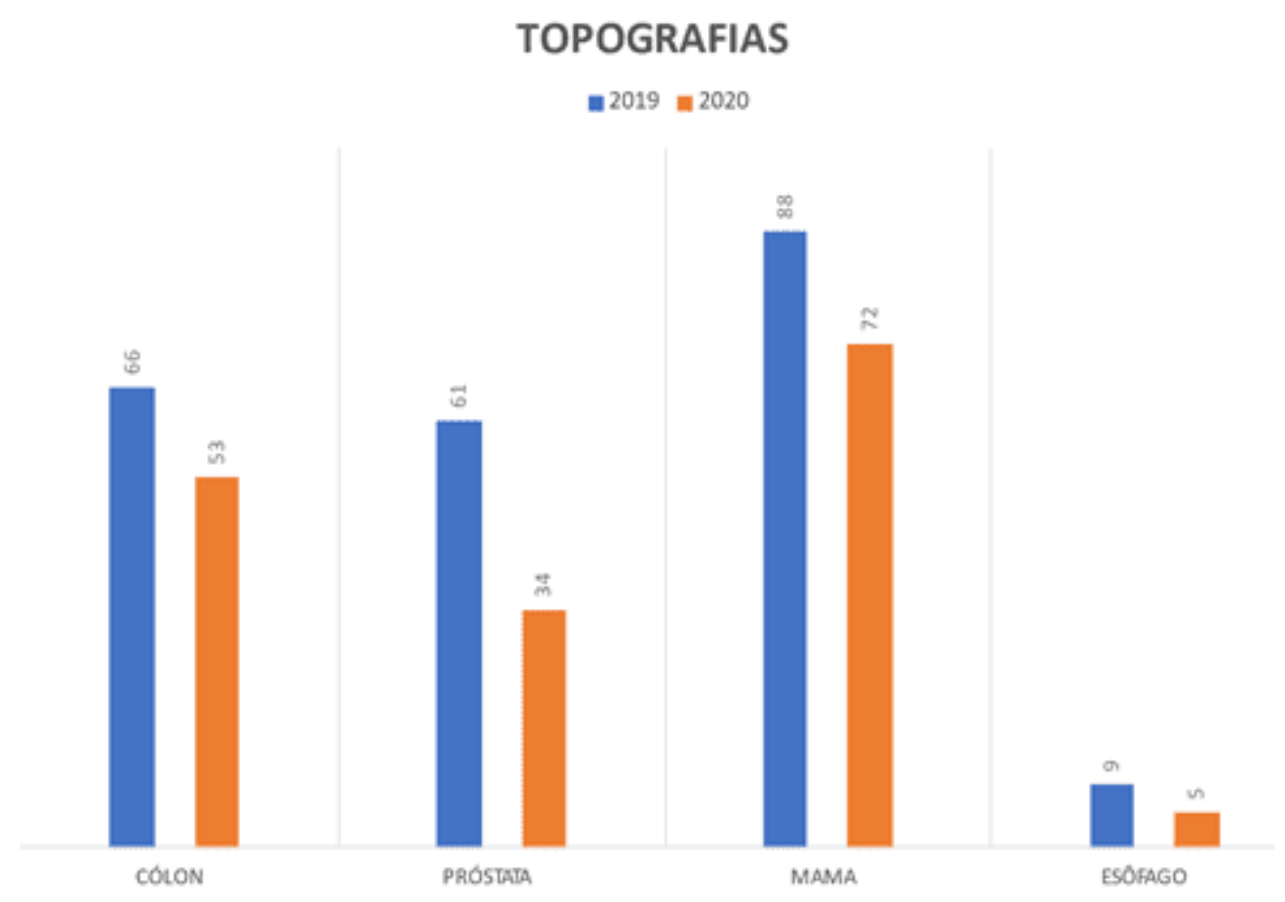

Quelle: Autoren.

Der Hauptrückgang war in Fällen, die ein invasives Verfahren erfordern würden, und in Fällen von Sedierung des Patienten. Andere Arten, wie Hautkrebs, nahmen im gleichen Zeitraum nicht ab. 
Die chirurgischen Blöcke der meisten Krankenhäuser beschränkten auch elektive Operationen, da Betten, insbesondere Intensivbetten, frei gelassen werden mussten.

Mit den Auswirkungen der Schließung vieler Kliniken und Kliniken in den frühen Stadien der Pandemie gab es einen großen Einfluss auf diese Diagnosen. Neben der Angst, dass Patienten ihre Häuser verlassen, war dies auch einer der Faktoren, die zu diesem Rückgang beitrugen.

Diese Studie hat einige Einschränkungen. Erstens wurden nur die Auswirkungen der Blockade auf die Diagnose in einer Gruppe von Labors bewertet, obwohl es sich um einen großen Dienst handelt. Die Ergebnisse der vorliegenden Studie sind daher nicht unbedingt auf andere Zentren übertragbar, so dass multizentrische Studien notwendig wären, um diese Ergebnisse auf nationaler Ebene zu validieren. Es ist möglich, dass dies zu einem Selektionsfehler führte. Diese Methode der Patientenauswahl war jedoch für alle untersuchten Zeiträume identisch, was eine Vergleichbarkeit ermöglichte.

\section{ENDGÜLTIGE ÜBERLEGUNGEN}

Zurück zur richtigen Frage: Wie hat sich die Pandemie auf die Diagnose neuer Krebsfälle in der Region ausgewirkt, die von einem Pathologiedienst in Südbrasilien abgedeckt wird? Durch die vorgestellte Studie konnte der Schluss gezogen werden, dass die daten, die in den Berichten von Patienten analysiert wurden, die in der Infolaudo-Gruppe gesehen wurden, zeigten, dass die Diagnose einiger Krebsarten in der Anfangsphase der Pandemie im Jahr 2020 mit dem analysierten Zeitraum von 2019 abnahm, was zeigt, dass die Pandemie diese Art von Diagnose beeinflusste. Es gab auch einen starken Rückgang der Anzahl der Biopsien und chirurgischen Proben, die in diesem Zeitraum erhalten wurden. Diese Erklärung kann multifaktoriell sein und durch die Schließung von Diagnosediensten wie Kliniken und Krankenhaussektoren sowie durch die Angst vor Patienten, die medizinische Versorgung suchen, beeinflusst werden. 


\section{VERWEISE}

ALLGAR, V. L.; NEAL, R. D. Delays in the diagnosis of six cancers: analysis of data from the National Survey of NHS Patients: Cancer. Br J Cancer, 2005.

AMBUSAIDI, A.; AL-BALUSHI, S. Educação em Saúde no Sultanato de Omã. In: Taylor, N. et al. (ed.). Educação em Saúde em Contexto. Editores Sense. 2012.

ASCO. American Society of Clinical Oncology. COVID-19 Patient Care Information. 2020. Disponível em: https://www.asco.org/asco-coronavirus-information/careindividuals-cancer-during-covid-19. Acesso em: 26 de jun. de 2020.

GREEN, T. et al. Cancer detection in primary care: insights from general practitioners. Ir. J Cancer, 2015.

HARFORD, J. B. Breast-cancer early detection in low-income and middleincome countries: do what you can versus one size fits all. Lancet Oncol, 2011.

INCA. Instituto Nacional de Câncer. Estimativa 2020: incidência de câncer no Brasil. Rio de Janeiro: INCA, 2020.

JONES, R. et al. Alarm symptoms in early diagnosis of cancer in primary care: cohort study using General Practice Research Database. BMJ, 2007.

KAUFMAN, H. W. et al. Changes in the Number of US Patients With Newly Identified Cancer Before and During the Coronavirus Disease 2019 (COVID-19) Pandemic. JAMA Netw Open, 2020.

LONDON, J. W. et al. Effects of the COVID-19 pandemic on cancer-related patient encounters. JCO Clinical Cancer Inform, 2020.

MACLEOD, $U$. et al. Risk factors for delayed presentation and referral of symptomatic cancer: evidence for common cancers. Br J Cancer, 2009. 
MARINGE, C. et al. The impact of the COVID-19 pandemic on cancer deaths due to delays in diagnosis in England, UK: a national, population-based, modelling study. Lancet Oncol, 2020.

VOSE, J. M. Delay in Cancer Screening and Diagnosis During the COVID-19 Pandemic: What Is the Cost? Oncology (Williston Park), 2020.

WHO. World Health Organization. Facts about cancer. 2016. Disponível em: www.who.int

Veröffentlicht: November 2021.

Genehmigt: November 2021. 